\title{
Theoretical and Experimental Investigations of Dynamics of the Flexible Rotor with an Additional Active Magnetic Bearing
}

\author{
Dorota Kozanecka
}

Additional information is available at the end of the chapter

http://dx.doi.org/10.5772/51113

\section{Introduction}

The conversion of energy in rotating machines is accompanied by phenomena that cause the additional dissipation of energy, affect technological processes, lower the endurance of machine elements and sometimes cause damages. Among these phenomena there are synchronous vibrations and self-excited lateral vibrations of the rotor and momentary pitches of amplitude of the rotor-bearings-foundation system vibrations.

Among the machines used in power engineering, rotary machines (compressors, pumps, blowers and turbines) are the ones used most often. Recent studies have put emphasis on the dynamics of rotating machines. This allows us to minimise the vibrations of the machine both during the period of its construction and at the time of its operation. The growing demand for reliability of rotating systems (i.e. API code) makes it necessary to specify their vibration parameters (critical frequencies, separation margins, amplitude of synchronous vibrations, permissible unbalance, etc.).

The search for new solutions of bearing systems in modern rotary machines that have to satisfy special performance demands has resulted in interest in rotor active magnetic suspension systems. The application of magnetic bearings as a system of shaft suspensions gives supplementary, unparalleled in classical solutions, diagnostic capabilities [10-14]. There are, however, also high requirements concerning the control system of the shaft position. New solutions in bearing systems have been more and more frequently applied in modern rotating machines. These include magnetic bearings that enable the active control of rotor vibrations.

A machine with a rotor supported in magnetic bearings allows for: 
- enhancement of the general efficiency due to the lack of a mechanical contact between the journal and the bush, and thus, a low level of power loss in comparison with classical slide bearings characterised by the same load carrying ability, and due to elimination of an oil system and seals connected with it,

- maintenance of absolute cleanness of the working medium and a hermetic structure of the whole machine without the rotating end-piece of the shaft outside the casing in the case of an application of a high-speed electric engine integrated with the shaft,

- operation under high rotational frequencies, in a wide range of temperatures (from $160^{\circ}$ to $250^{\circ} \mathrm{C}$ ) in chemically aggressive environments or in vacuum.

An active magnetic bearing system is a qualitatively different technology in comparison with classical solutions and requires the co-operation of specialists from two branches of technology, as it is a combination of a mechanical system with an electronic automatic control system, which controls this mechanical system $[1,10]$.

A scheme in Figure 1 presents an active magnetic bearing as an automatic control system for one of the control axes - $y$. The voltage signal from the displacement transducer $U_{D T}$ is conditioned. A change in the position of the journal with respect to the reference position $\left(U_{\text {Ref }}-U_{D T}\right)$ activates the control current flowing through the bearing bush winding (electromagnet) in the electronic control system. This control results in a change in the electromagnet forces $F_{m}$ that brings the journal to the assigned position.

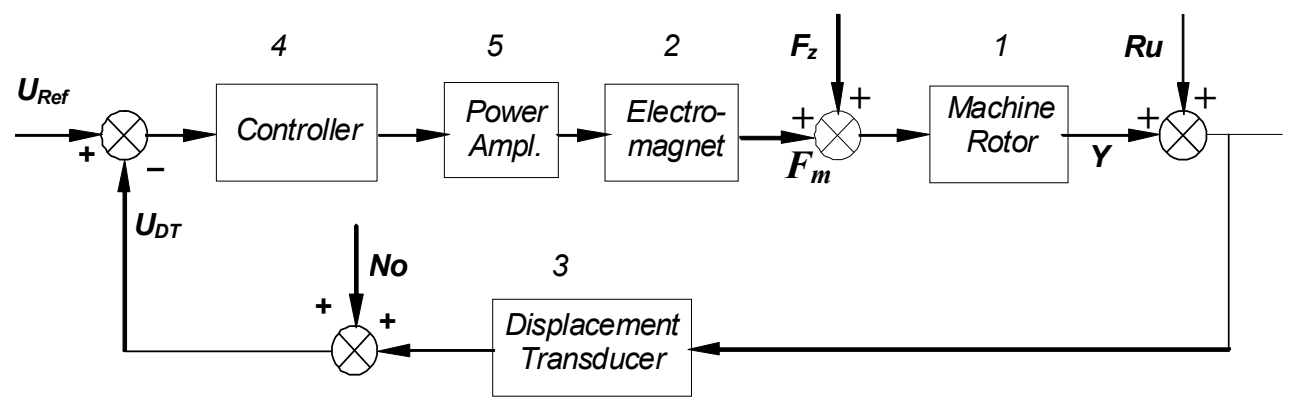

Figure 1. Scheme of the active magnetic bearing as an automatic control system (one of the control axes $-y)$.

A proper value of the current is generated by the power amplifier on the basis of the signal provided by the controller according to the assumed control algorithm as a function of the present position $y$ of the journal, measured by means of displacement transducers (Figures 1, 2).

Apart from disturbances connected with forces coming from e.g. unbalancing $\mathrm{Fz}$ and static loading forces $F_{\text {stat }}$, the bearing system is affected by accidental interference No (noise) introduced by displacement transducers and interference following from heterogeneity of the measuring path of the runout type $R u$. A level of these disturbances has a very significant influence from the viewpoint of stable operation of the system. A structure and algorithm of the applied controller have to ensure the system resistance to their effects. 
An active magnetic bearing comprises two distinct components:

- a bearing itself, and

- $\quad$ an electronic control system.

A radial bearing comprises a rotor on which ferromagnetic laminations are fitted. The rotor is held in position by four electromagnets placed equally around the rotor, normally at 45 degrees to the vertical axis (Figure 2). The position of rotor (1) is monitored constantly by sensors (3) that detect any deviation from the nominal position. Any such deviation results in a signal which, by means of electronic control system $(4,5)$, is compared to the reference value. The resulting signal increases or decreases the current flowing to electromagnets (2) and thus returns the rotor to its nominal position. Each electromagnet produces an attractive force acting on the rotor.

An increase in high-speed turbomachinery applications has led to a significant progress in the research of rotor dynamics in the last decade. The evaluation of the dynamic stability and response to unbalance has become a standard calculation procedure for all new turbomachinery designs.

In order to apply magnetic bearings to suspend rotors of real machines, proper design methods that account for a special character of their operation and that are adapted to the requirements the bearings are to satisfy are needed. There is a desirable tendency in rotating machines to design rigid rotors because of a relatively low level of vibrations in their whole operating range which is observed. This aim can also be achieved by using flexible rotors with auxiliary magnetic bearings whose adjustable characteristics may be used to exceed safely the critical frequency of the rotor - bearing system with low amplitude of vibrations.

This work presents an idea of maintaining a low level of vibrations in the whole operating range of the rotating system of the flexible rotor (including critical speeds) by using an additional active magnetic bearing where the biggest anticipated dynamic deflection of the rotor occurs. What is achieved is the momentary operation of an additional magnetic bearing in the rotating system before a high level of the amplitude, which corresponds to the critical frequency of lateral vibrations. This causes a qualitative change in the dynamic properties of the system. It also allows one to reach the nominal revolutions of the machine without the dangerous effects connected with exceeding the critical frequencies of the flexible rotor.

The realisation of the presented idea for the real rotating system requires a preparation of the theoretical and experimental methods of investigations and numerical simulations for the model of active magnetic suspension.

In this work the theoretical and experimental investigations of the digitally controlled active magnetic bearing and the theoretical and experimental investigations the flexible rotor dynamics carried out on the test stands with an auxiliary active magnetic bearing with a digital control system are discussed $[5,6,9]$. 


\section{Digitally controlled magnetic bearing}

Active magnetic suspension systems of machine rotors being built at present are equipped with digital control systems. Apart from a possibility of implementation of complex control algorithms, they provide also wide diagnostic possibilities resulting from an application of measurement techniques at different stages of the system design. Control systems of bearing responses decide about dynamic properties of the rotating system. Digital controllers allow for, e.g. a change in bearing dynamic properties during motion in different modes of the machine operation.

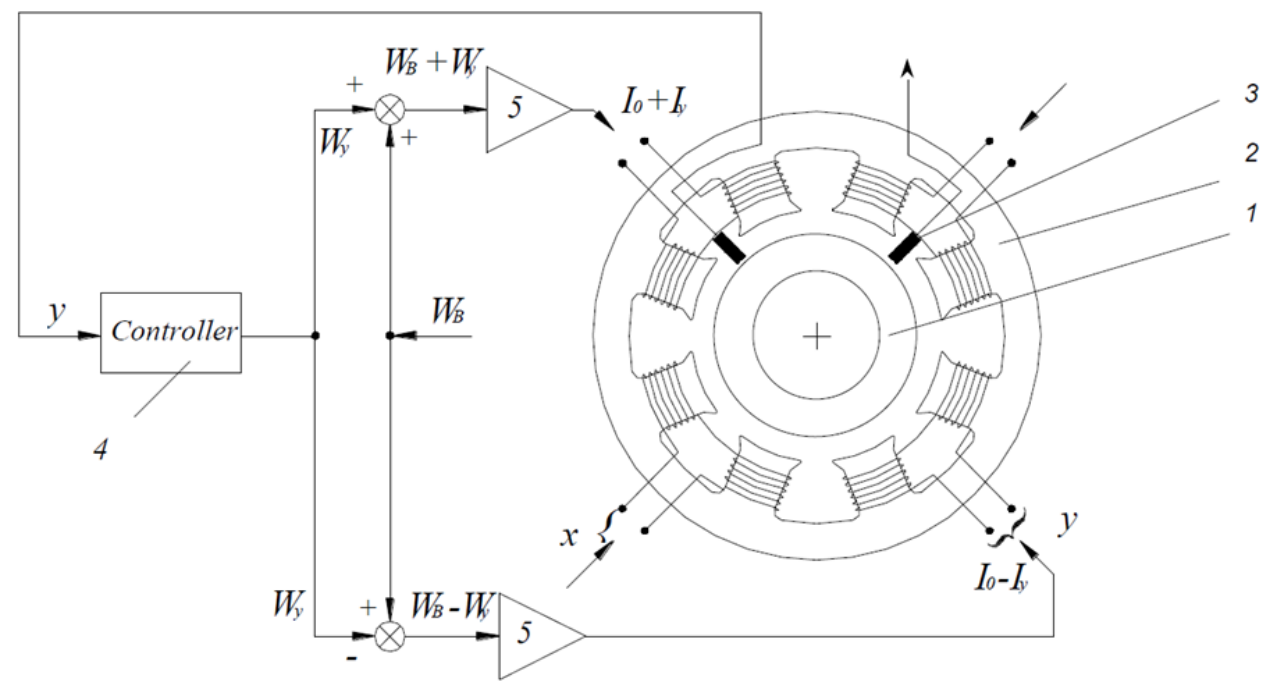

Figure 2. System of the digitally controlled magnetic bearing (one of the control axes - y).

The research on active magnetic bearing technology, including works on digital controllers and algorithms, actuators and magnetic bearing-rotor system dynamics, has been carried out for several years in the Institute of Turbomachinery of the Technical University of Łodz [4-9]. The mechanical structure of the built active magnetic bearing, consists of a journal and a bush with four pairs of electromagnets placed equally around the rotor. The position of ferromagnetic journal (1) with respect to bush (2) is controlled by means of eddy-current displacement transducers (3) made by Bently Nevada Corporation, with the diameter $d=8 \mathrm{~mm}$ and the static sensitivity $7.870 \mathrm{~V} / \mathrm{mm}$. They are mounted on two control axes $x, y$ that are perpendicular with respect to each other and displaced by the angle $45^{\circ}$ with respect to the journal axis. The control axes interact with respective pairs of electromagnets (Figure 2).

Each pair of the bush electromagnets of the journal bearing interacts with a digitally controlled power amplifier with a variable pulse width PWM (5). The control pulse-width modulation $W_{y}$ and $W_{x}$ is counted by controller (4) on the basis of measurements of the position $y$ and $x$ of the journal, respectively. The control current that supplies the windings 
of individual electromagnets is an explicit function of this modulation. For the journal magnetic bearing, the differential control is used and it requires a generation of the bias current $I_{0}$. To obtain the bias current $I$ o for the designed bearing, the pulse-width modulation $W_{B}$, which determines its operating point on the characteristics, is used.

\section{Numerical model of the magnetic bearing system}

The basic assumption while developing the numerical model of the bearing system was to offer a tool that allows for tuning the parameters of its controller and for carrying out the investigations of the designed system dynamics in a wide range $[4,14]$. The condition to be met during the realisation of the idea of the numerical simulation of the bearing was to reproduce the algorithm of operation of the real bearing actuating system structure, and the measurement and control elements applied in this real system in the model. The fulfilment of this requirement has guaranteed the correctness of the operation of the model and feasibility of its design.

A general scheme of the system that has been employed in order to develop the numerical model is presented in Figure 3a. In this model, a motion of the mass $m$ concentrated in the geometrical center of the journal is analyzed.

A controller used in the system controls both the axes $x$ and $y$. For each axis, differential control with a programmed value of the pulse-width modulation of the so-called $W_{B}$ base has been applied. Figure $3 \mathrm{~b}$ shows system of the position of the journal with respect to the bearing bush: EM1, EM2 - bush electromagnets interacting with the axis $y, E M 4, E M 3$ - bush electromagnets interacting with the axis $x, F z$ - rotating vector of residual unbalancing, $F_{m x}-$ electromagnet force acting along the axis $x, F_{m y}$ - electromagnet force acting along the axis $y$, $F_{g}+F_{\text {stat }}-$ forces of gravity and static load.

a.

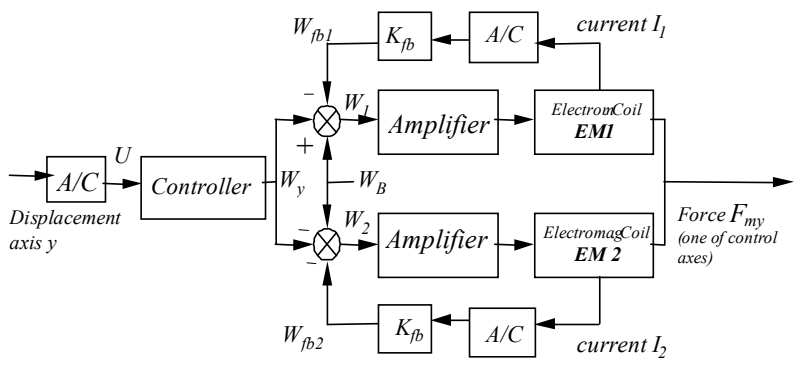

$b$.

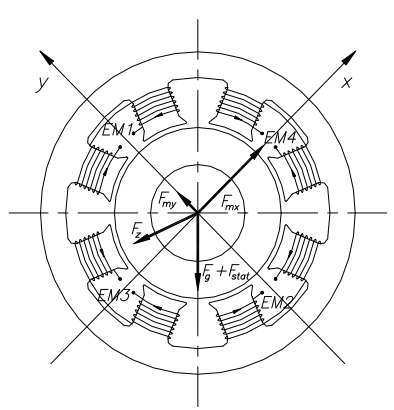

Figure 3. a. - Diagram of the model conception, b. - Distribution of forces (one of the control axes - y)

An idea of this model assumes a possibility of numerical simulations of dynamic properties of the system, owing to three defined levels of data connected with the bearing system structure, namely: 
- physical parameters of the bearing: mass supported in the bearing, radial clearance of the magnetic bearing, initial position of the journal with respect to the bush;

- parameters of the controller structure and the control system structure: boundary values of the pulse-width modulation $W_{\min }, W_{\max }$, and $W_{B}$ which determines the operating point on the characteristics, feedback coefficient $K_{f b}$

- $\quad$ parameters of the actuating systems: frequency of control pulses $f_{P W M}$, power amplifier supply voltage $U z$, inductance of the actuating system $L$, resistance of the actuating system $R$, value of the bearing constant $K$.

The model gives also a possibility of analysis of permissible levels of disturbances $F z, R u$, No that provide a proper margin of the bearing system stability for a given type of the controller. Thus, in a sense, it constitutes the synthesis of a robust controller.

The numerical procedures representing actual characteristics of the actuator were developed and verified, and then applied in the simulation model of the bearing. This allows for modelling the magnetic bearing system quickly and accurately. The numerical simulation of the active magnetic bearings system by means of the Hewlett-Packard HPVEE software was elaborated.

In the designed digital control system of a journal active magnetic bearing, the suitable software that allows for investigations of the pulse-width modulation - control current characteristics that determine the bearing system properties while its actuators are built, has been applied.

\subsection{Numerical characteristics pulse-width modulation - control current}

Each pair of the bush electromagnets of the built journal bearing interacts with a digitally controlled power amplifier with a variable pulse width PWM. The control pulse-width modulation $W$ is counted by the controller on the basis of measurements of the position of the journal with respect to the bush. The control current that supplies the windings of individual electromagnets is an explicit function of this modulation $[4,9]$.

Power transistors and discharging diodes have been used to build the amplifiers (Figure 4). They are characterized by specified values of operating parameters. The properties of transistors and diodes, as well as the winding inductance and resistance of a given pair of poles, determine the parameters of the whole bearing actuator path, in which control current is generated.

The dynamics of changes of the current in windings is strictly dependent on the force generated by electromagnets in the gap between the journal and the bush and it affects directly the dynamics of the mass suspended in the bearing. The knowledge of actual characteristics of the actuator paths is an important factor in designing the structure and the algorithm of the controller, whose task is to ensure stable operation of the bearing.

According to these assumptions, a development of the program that allows for numerical simulation of the theoretical equations describing the phenomena occurring in the actuator 
electric circuit versus time (i.e., in subsequent periods of control pulses) for nominal parameters of the power amplifier elements, electromagnet windings, supply voltage and control frequency, was required.
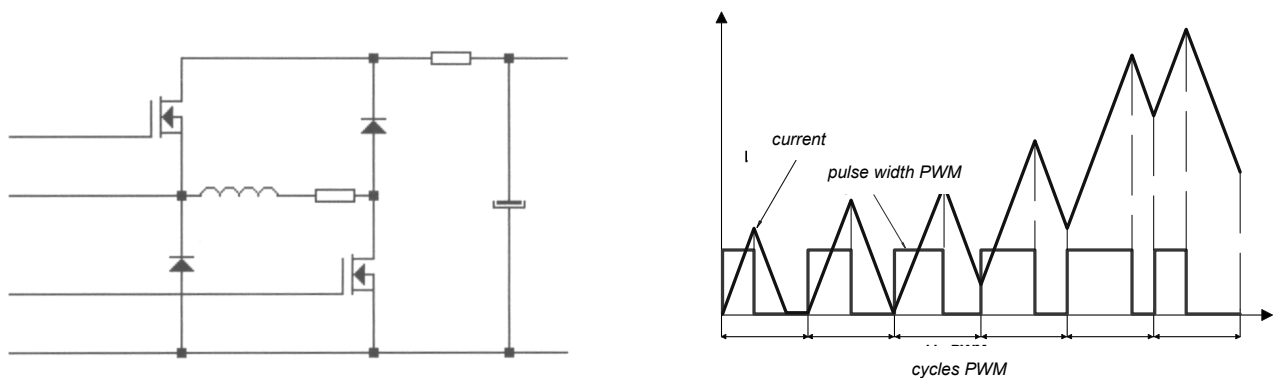

Figure 4. Power amplifier circuit and an idea of current changes in the bearing

An operation of the actuator was analyzed. Each cycle of the control pulse PWM of a given frequency $f_{P W M}$ and a pulse-width modulation $W$ forces two modes of the amplifier operation, namely:

- $\quad$ charging, whose duration is equal to $t_{\text {char }}=W / f_{P W M}$. Elements of the amplifier circuit operate then in the conduction mode,

- discharging, whose duration is equal to $t_{\text {dischar }}=(1-W) / f_{P W M}$. Elements of the amplifier circuit operate in the lockout mode.

For a given pulse-width modulation, a value of the control current $I_{a v} i$ that generates the bearing magnetic force results from its averaged value in the $P W M$ cycle and has been expressed by relation (1):

$$
I_{a v i}=W_{i} \frac{I_{0 i}+I_{\max i}}{2}+\left(1-W_{i}\right) X_{i} \frac{I_{\max i}+I_{0 i+1}}{2}
$$

$I_{0 i}-$ initial current for the $i-$ th cycle of the amplifier charging.

$I_{0 i+1}-$ initial current for the $i+1-t h$ cycle of the amplifier charging.

The coefficient $X_{i}$ occurring in formula (1) determines a ratio of the discharging time for a given cycle to the time during which the power amplifier electronic elements cause that the control current value diminishes to zero. In the cycles in which the current discharges to $I_{\min i}$ $=I_{0 i+1}$, the coefficient $X_{i}=1$.

Individual operation sequences of the digitally controlled pulse power amplifier and the windings powered by it have to be analyzed. Numerically simulated sequences of the ideal actuator operation enable one to generate model characteristics, which are the basis for evaluation of an influence of changes in values of individual parameters of the actual system on their shape. 
Numerical calculation procedures that allow for the generation of instantaneous and mean time histories of control currents in the assumed PWM cycles, for the following actuator parameters: amplifier supply voltage $U_{z}$, inductance of the bearing bush winding $L$, resistance of the power amplifier circuit $R$, frequency of control pulses $f_{P W M}$ as a function of values of the pulse-width modulation coefficient $W$ of the control pulse, have been developed.

In Figure $4 \mathrm{a}$ and $\mathrm{b}$ exemplary pulse-width modulation - control current characteristics of the bearing actuator system calculated for selected values of its parameters are shown.

Nominal, experimentally identified values of the parameters of the actuators assumed in the calculations were as follows:

- $\quad$ inductance of the electromagnet winding $L=80 \mathrm{mH}$,

- $\quad$ resistance of the power amplifier circuit $R=3.1 \Omega$.

- supply voltage of the power amplifier $U_{z}=80 \mathrm{~V}$,

- frequency $f_{P W M}=1667 \mathrm{~Hz} \Rightarrow T_{P W M}=600 \mu s$.

a.

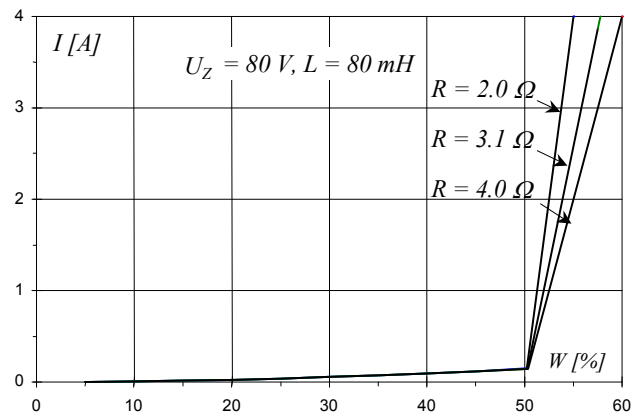

$b$.

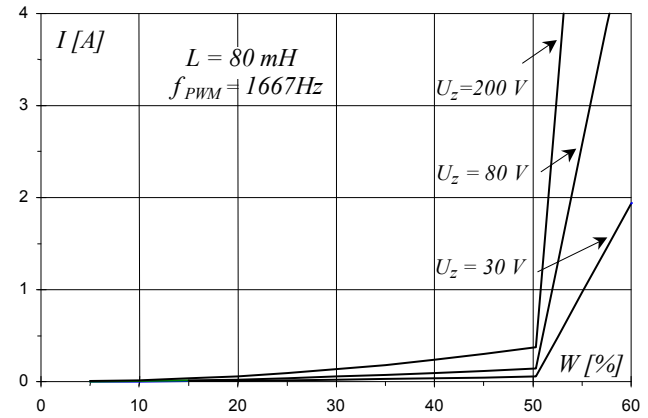

Figure 5. Pulse-width modulation - control current characteristics of the bearing actuator a. - as a function of the amplifier circuit resistance, b.- as a function of the power amplifier supply voltage

The characteristics were calculated for experimentally identified parameters of the actuator systems in order to enable their verification for a real object at each stage of the calculations.

To show a tendency of changes, the characteristics generated for bigger and smaller parameters than the nominal ones have been presented in the figures as well. This allows one to forecast and evaluate a possibility of introducing changes in the values of parameters of the designed bearing system actuator. It is of great practical importance.

From the characteristics shown in Figure 5a and b, it follows that for the structure of the power amplifier under investigation and for the real parameters of bush windings, the actuator has to operate under pulse-width modulations $W>50 \%$ in order to ensure a suitable load capacity. 
For a pulse-width modulation $W>50 \%$, the dynamics of changes in the control current depends on resistance in the power amplifier circuit. The total value of resistance includes electromagnet winding resistance and dynamic resistance of the amplifier electronic elements in subsequent cycles of its pulsating operation, i.e. during charging and discharging (Figure 5a). An increase in the dynamics of the current changes in the bearing system under consideration, i.e. for given, actual parameters of the bush windings and the power amplifier, can be achieved through an increase in the value of the power amplifier supply voltage $U z$ (Figure $5 b$ ).

\subsection{Experimental characteristics pulse-width modulation - control current}

In order to identify the properties of each actuator path, suitable algorithms, software for a microprocessor of the measurement-control card and a host computer of the bearing system have had to be prepared. Independent auxiliary procedures that allow for experimental testing the actuator paths of the bearing have been developed. These procedures form a program for operation of the measurement-control card, which is the main element of the bearing digital control system [11].

An active magnetic bearing system comprises actuators, which are realised in the form of windings of individual pairs of bush electromagnets that interact with digitally controlled power amplifiers. A scatter of real values of electric parameters of the winding for individual pairs of electromagnets, to which a technological scatter of the amplifier structure elements is added, will introduce the asymmetry of properties for the control axes.

From the point of view of simplification of the structure designing and the controller parameter tuning in order to obtain the system stable operation, individual bearing actuators should be characterised by the symmetry of properties. A fulfilment of this condition will allow for making the mechanical characteristics of the bearing system independent of the actuator properties for each axis.

Control systems of the bearing response are a very important part of a machine with active magnetic bearings because they decide about the dynamic characteristics of the rotor. In order to design and build a control system fulfilling the requirements of the object under investigation, the knowledge of its real model is needed.

An active journal magnetic bearing with a digital control system has been built according to the presented idea. A new concept of control systems has been elaborated. It allows one to program the control of the bearing actuator characteristics. To achieve this goal, a programming procedure for the control processor of the bearing has been developed. The programmed testing procedure of an actuator allows one to reach experimental characteristics for its various parameters (frequency and width $P W M$, power supply, air gap between the rotor and the stator) for each control axis [5].

The experimentally determined pulse-width modulation $W$ - control current I characteristics show differences in the bearing properties for two control axes $x$ and $y$ (Figure 6a). As a 
result, they render the asymmetry of mechanical properties of the system for these axes when the feedback loop is closed and the bearing control system is turned on.

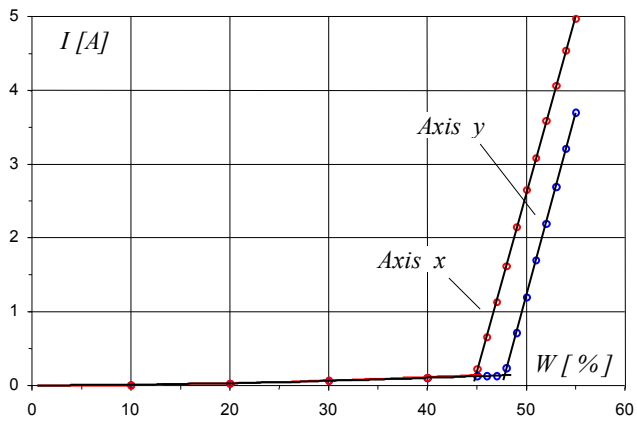

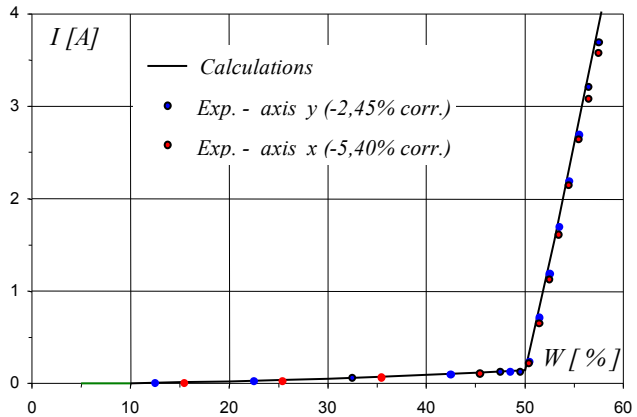

Figure 6. a. - Experimental pulse-width modulation $W$ - control current $I$ characteristics of the bearing actuator systems before programmed correction, $\mathrm{b}$. - Theoretical and experimental characteristics after programmed correction.

For the given parameters of a real bearing system, there is always a scattering of properties of the actuating system for each pair of electromagnets. The developed procedure enables one to tune these characteristics in order to obtain the symmetry of operation of actuating systems for each pair of electromagnets and for each bearing control axis $y$ and $x$.

An alternative to compensation for the asymmetry of bearing actuator properties by designing a proper structure and algorithm of the controller is to develop a method of its programmed correction. On the assumption that the source of the identified asymmetry lies in a scatter of actuator parameters, a correction method that leads to minimisation of the effect of the scatter of their characteristics for each bearing control axis has been proposed.

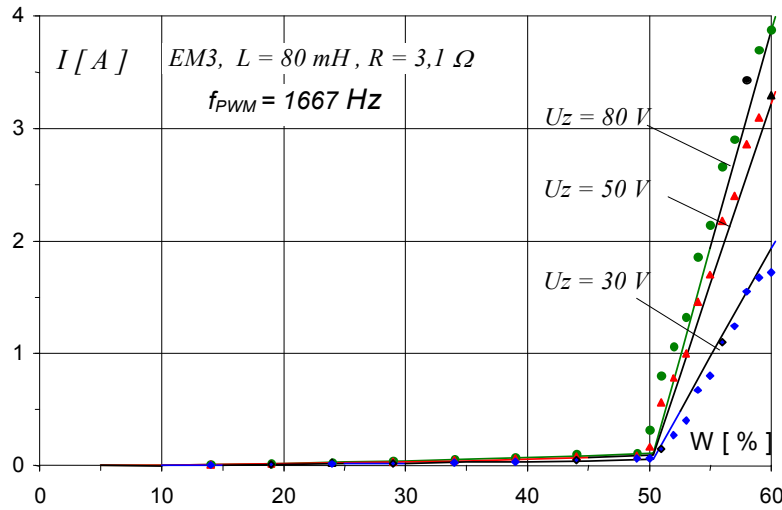

Figure 7. Theoretical (solid line) and experimental (dots) pulse-width modulation - control current characteristics of the bearing actuator systems after the programmed correction for various values of the power amplifier supply voltage 
From the pulse-width modulation - control current characteristics that were experimentally determined (Figure 6a), it follows that the dynamics of the current changes increases already at the pulse-width modulations $\mathrm{W}<50 \%$ in an actual actuator. These phenomena are connected with the structure of control systems, power amplifier transistor gates and they need a necessary correction. The theoretical characteristics are used to evaluate a scatter of properties of real actuator paths of the bearing. This evaluation allows one to introduce necessary programmed corrections of characteristics of real actuator systems to achieve the convergence between the characteristics determined experimentally and those calculated numerically [6].

This correction results in obtaining the symmetry of properties of individual actuators of the designed bearing which interact with the bush electromagnet windings EM1, EM2, EM3, EM4 (Figure 6.b).

After this correction, pulse-width modulation - control current characteristics of actuator paths of the bearing have been determined experimentally. The results of experimental investigations and numerical simulations have exhibited a good convergence. Figure 7 shows these characteristics for the bearing actuator path interacting with the axis $x$ - EM3 for different values of the power amplifier supply voltage.

The proposed way of the programmed correction of the characteristics has been verified experimentally and carried out for a real bearing structure $[4,5,6]$.

\subsection{Numerical calculations and verifications}

In the analysis of model investigation results, the same quantities have been chosen as those recorded in a real magnetic bearing system, namely: pulse-width modulation, displacement, static equilibrium position, journal trajectory, phase portrait, Bode's plot, etc. It has enabled their direct verification with the experiment. The verification has been carried out on the test stand with the built radial bearing, whose part is presented in Figure 8.

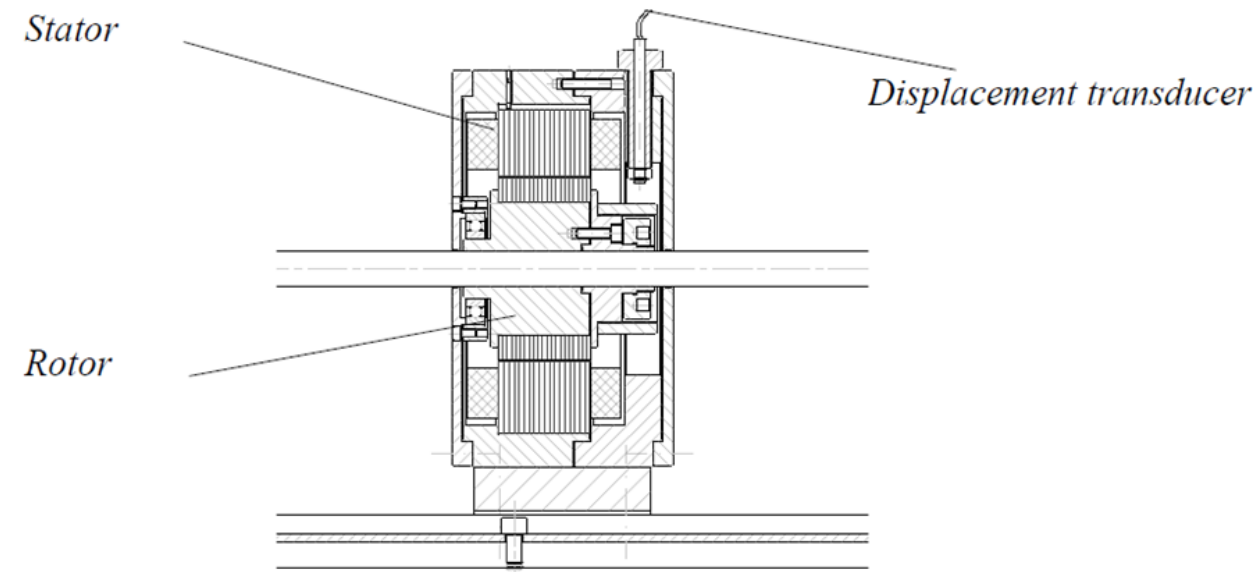

Figure 8. Structure of the built radial bearing 
The investigations of the bearing dynamics comprise three basic modes of its operation:

- suspension of the journal in the bush,

- start-up,

- operation under constant rotational frequency.

Some selected test results for the first mode of the system operation are presented.

The nominal values of parameters that have been assumed in the model calculations are as follows:

- $\quad$ power amplifier supply voltage $U z=80 \mathrm{~V}$

- frequency of control pulses $f_{P W M}=1667 \mathrm{~Hz} \Rightarrow T_{P W M}=600 \mu \mathrm{s}$

- inductance of the electromagnet winding $L=80 \mathrm{mH}$

- $\quad$ resistance of the power amplifier circuit, including the windings $R=3.1 \Omega$

- bearing constant $K=2.410^{-5} \mathrm{Nm}^{2} / A^{2}$

The bearing constant $K$, as well as the power amplifier circuit resistance and inductance, $R$, $L$, have been determined experimentally through suitable measurement procedures for the real structure of the bearing system, whose actuators operate at the above-mentioned values of the supply voltage $U z$ and the frequency $f_{P W M}$.

In order to present the diagnostic capabilities of the model developed and to indicate a convergence of its operation with the real system, the suitably selected characteristics calculated numerically for the defined nominal parameters and those recorded for the real bearing system have been shown in Figures 9 - 13.
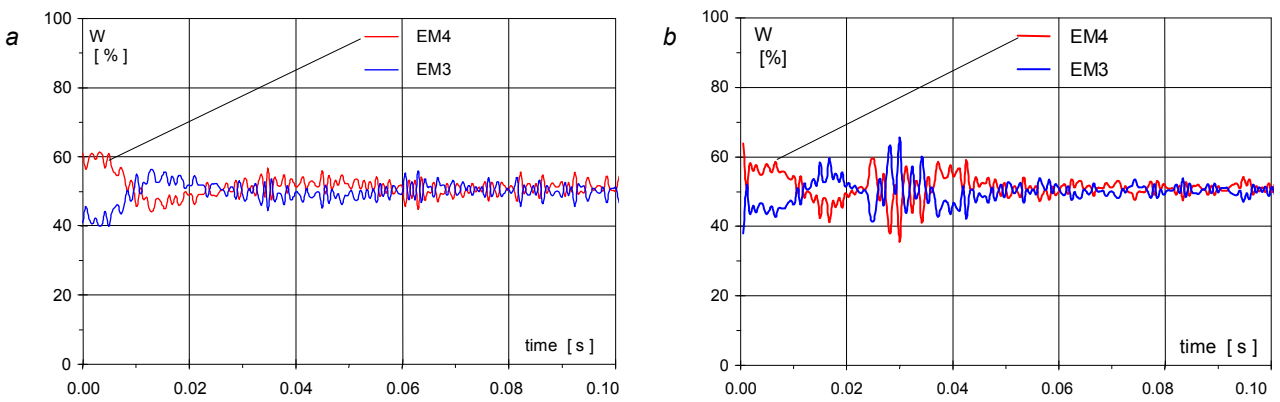

Figure 9. Changes in the pulse-width modulation during the suspension of the journal in the bush for the control axis $\mathrm{x}$ (electromagnets: top - EM4 and bottom - EM3) - a. - numerical simulations, b.experimental results

For one control axis during given sampling time, pulses with the pulse-width modulation $W$ proportional to the signal produced by the controller are calculated on the basis of the displacement measurement of the journal with respect to the bush. After its comparison with the assigned pulse-width modulation that determines the bearing $W_{B}$ operating point and the pulse-width modulation that corresponds to the value of the current flowing in the top and bottom electromagnet winding $W_{f b}$, the pulse-width 
modulation $W_{f b}$ is realized through the application of an additional feedback loop with a possibility of programmed assigning the value of the feedback coefficient $K_{f b}$.

In Figs. $8 \mathrm{a}$ and $\mathrm{b}$ changes in the pulse-width modulation as a function of time in the mode of suspension of the journal in the bush for the windings of the top electromagnet EM4 and the bottom one EM3 interacting with the axis $\mathrm{x}$ are shown. The data recording for the real bearing structure was carried out by means of the developed PRDP procedure. The presented calculation results and the recorded results refer to the same parameters of the controller. A good convergence of the results has been obtained, which confirms reliability of the model built.

On the basis of the calculated pulse-width modulations, the bearing actuators generate currents that control the windings of corresponding bush electromagnets. The experimentally verified procedures that represent the real actuator operation for the assigned parameters have been employed in the algorithm of the model operation.
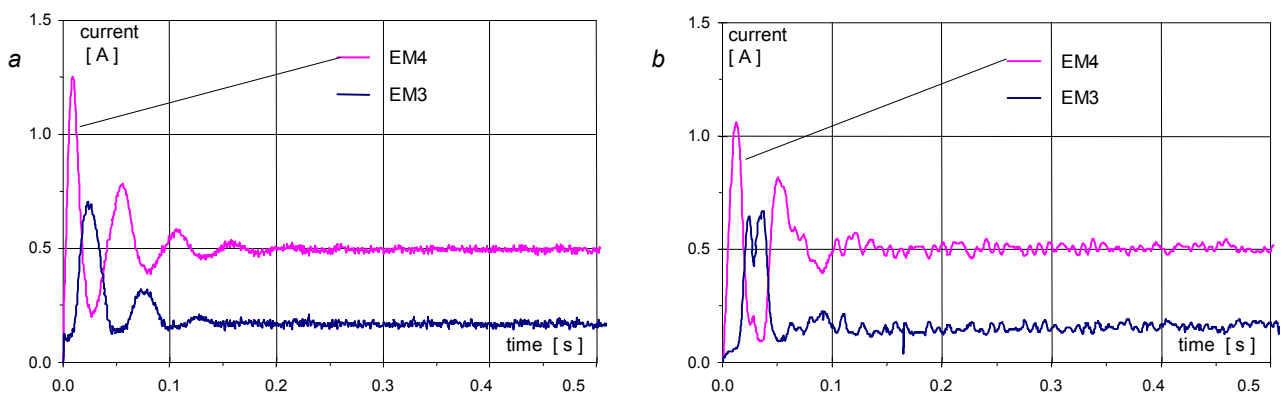

Figure 10. Currents in the windings of the top EM4 and bottom EM3 electromagnet - axis $x$ a. - numerical simulations, b. - experimental results

For the real structure, the values of correction, indispensable to obtain the symmetry of bearing properties, have been introduced for each pair of windings for both the control axes $x$ and $y$.

In Figures $10 \mathrm{a}$ and $\mathrm{b}$ changes in the current versus time are shown in the mode of suspension of the journal in the bush for the electromagnet top windings EM4, EM1 and bottom windings EM3, EM2, interacting with the corresponding control axes $x$ and $y$. The control currents in the bush top and bottom winding along each control axis are timevariable and are a function of the change in the journal position with respect to the bush until the equilibrium position is achieved.

Figures 11a and $\mathrm{b}$ show changes in the displacement of the magnetic bearing journal at the moment an active magnetic bearing is activated for both the control axes $x, y$ for the real system and its simulation model, respectively.

In Figures 11 and 12 an effect of the bearing system operation in its first operation mode in the form of the calculated and recorded changes in the position of the journal with respect to 
the bush as a function of time is presented for both the control axes and for the orbit composed of these displacements.
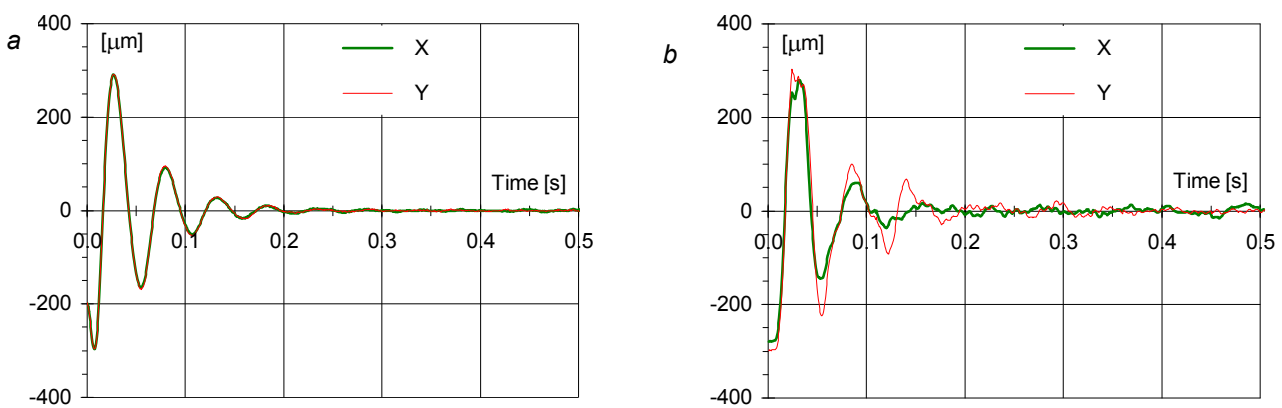

Figure 11. Displacement of the rotor a. - numerical simulation, b.- experimental results
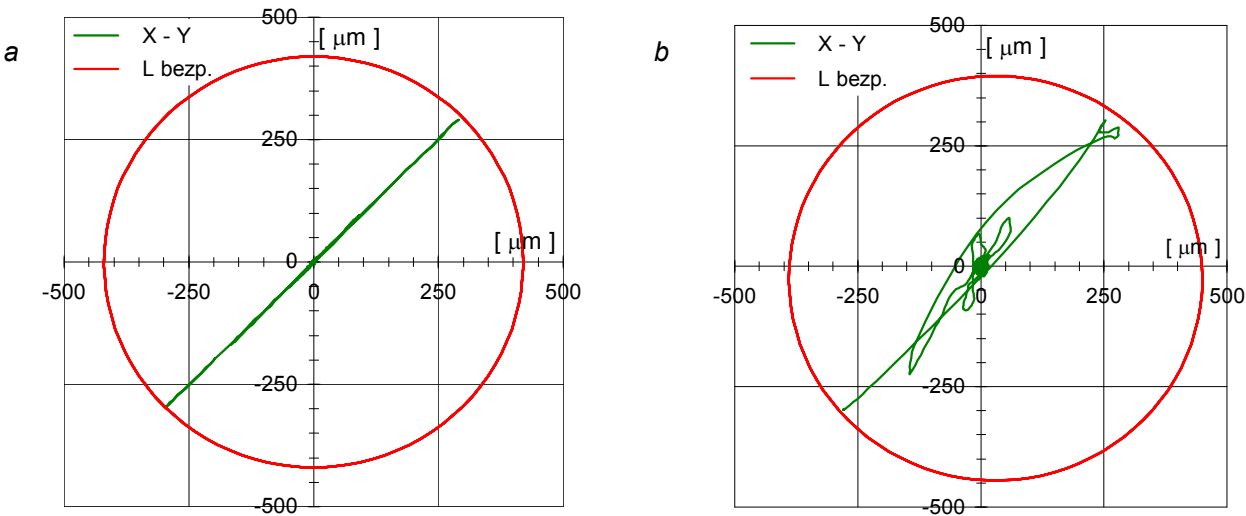

Figure 12. Orbit of the rotor a. - numerical simulations, b. - experimental results

At the ideal symmetry of the properties of the actuators for each control axis, which is assumed in the model, symmetrical time histories of the journal displacement for both the position control axes $x(t)=y(t)$ are obtained - Figure10 a. In the real system, slight differences in the time history of the journal displacement occur at the moment of its startup in comparison with the model. These differences result from: non-ideal geometry of the journal, asymmetry of the arrangement of the position sensors with respect to the journal or asymmetry of the journal position with respect to the bush (Figures 11b, 12b).

The character of the changes in the journal position as well as the time that passes from the moment the control system is activated up to the moment in which stable suspension of the mass supported in the magnetic bearing is achieved are the same for the model and for the real structure under investigation.

In Figure 13 changes in the magnetic force generated in the bearing system during suspension of the journal in the bush versus time are shown. The characteristics is obtained on the basis of the simulations. 


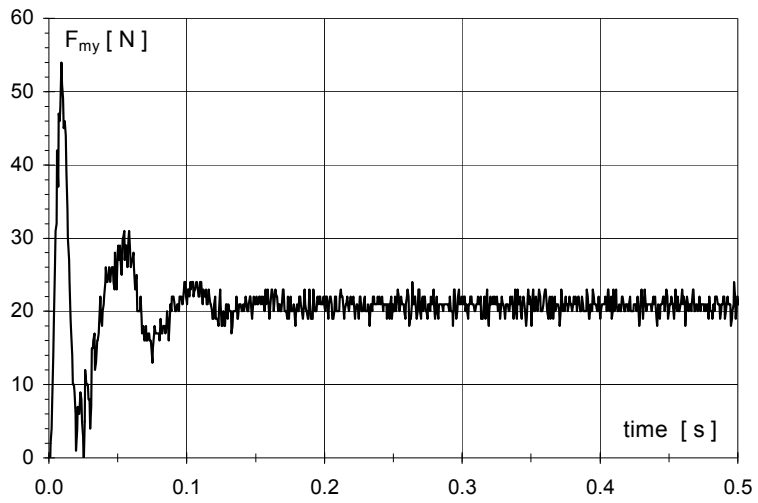

Figure 13. Magnetic force $F_{y m a g}$ generated in the bearing system - axis $y$

The agreement between the simulation investigations and the real bearing structure investigations has confirmed that the simulation model developed is a reliable tool that can be employed in designing a bearing system and in forecasting the tendencies of changes in its operation under the assigned level of disturbances and assigned excitations.

Their comparison indicates the feasibility of the simulation model in practical applications.

In order to apply simulation investigations in practice, their verification for an actual object is needed. The basic assumptions connected with the developed simulation model of an active magnetic bearing system are presented. The simulation and experimental investigations have been verified on the test stand for a digitally controlled journal active magnetic bearing. The results discussed confirm a convergence between the operation of the model and real bearing.

A reliable theoretical model that allows for analysis of the bearing dynamics under hypothetical, extreme loads reduces the designing time and enables one to minimize errors that can occur at the system prototype start-up.

The tool allows for testing different variants of the magnetic bearing system operation, which has been confirmed by the experimental investigations conducted in a wide range. A diagnostic capability of the non-linear numerical model of the magnetic bearing allows to develop a method for the identification of dynamical parameters: stiffness and damping $[8,9]$.

\section{Concept of the method for the identification of dynamic parameters}

In the proposed measurement method, a dependence of the vector of the resultant magnetic force acting on the machine shaft as a function of the journal position in the magnetic radial bearing and the currents that flow through the windings of actuators (electromagnets) is employed. The magnetic bearing response vector is a sum of the forces generated by bearing 
electromagnets and alters in each control cycle PWM [5,9]. The value of the magnetic response component $F_{X m a g}$ for one control axis is related to the measured mean values of the current controlling the electromagnets $I_{X T}, I_{X B}$ in a given control period and the values of the magnetic gaps ${ }_{X X T}, S_{X B}($ top - index $T$, bottom - index $B$ ). The values of the magnetic gaps are found on the basis of measurements of instantaneous positions of the journal with respect to the centre of the bush of the known clearance. The magnetic response component Fxmag for the axis $X$ is determined by the following relationship:

$$
F_{X \text { mag }}=K_{X T} \frac{I_{X T}^{2}}{s_{X T}^{2}}-K_{X B} \frac{I_{X B}^{2}}{s_{X B}^{2}}
$$

Equation (2) holds on the assumption that the linear dependence of the magnetic flux on the induction is maintained. It means that the bearing operates according to this part of the characteristics that is distant enough from the state of magnetic circuit saturation, when the induction does not exceed $50 \%$ of the saturation induction for the core material. The value of the constant $K$ depends on electromagnet design parameters and can be calculated theoretically [1]. However, in the actual design of the journal bearing, the constants $K_{x T}, K_{x в}$ $K_{Y T}, K_{Y B}$ can differ slightly for each pair of electromagnets of the bush. In order to increase the accuracy of the proposed measurement method, the constant values are verified experimentally for each electromagnet and their real values are taken into account in the calculations [8]. If the journal motion parameters are known and the magnetic response force is determined by an indirect method, it is possible to find the bearing dynamic parameters that relate the magnetic response force to the journal motion parameters $[5,9]$.

An analysis of the system response to synchronous excitation points out to the fact that the bearing magnetic response force $F_{x m a g} F_{y m a g}$ is proportional to the excitation force amplitude $F_{z}$ in the whole range of frequency of rotations. It allows for the identification of equivalent dynamic coefficients of the bearing. In the proposed method a non-linear analysis of the simulation model in the assumed range of rotational frequency changes of the rotating mass of the bearing, for the excitation with the rotating vector of unbalance $F z$, is required. For each assumed value of the frequency of rotations, the value of unbalance $F z$ is chosen in a such a way that the forced vibrations of the bearing rotating mass have the same value of amplitude in each control axes $x, y$. For the assumed frequency values $n$, the time histories of the following quantities are recorded:

- excitation unbalance force $F z(t)$,

- variable components of displacement of the rotor mass along the respective control axes $x_{a c}(t), y_{a c}(t)$,

- velocity components of the rotor mass in the control axes $v x(t), v y(t)$,

- $\quad$ variable components of the magnetic response forces for the control axes $F_{x m a g} a c(t), F$ ymag $a c(t)$.

These time histories are non-linear, as no simplified assumptions have been introduced into the model for the relations between the force, current and displacement. In the final stage of the proposed method, a relation between the linear stiffness and damping coefficients and 
the bearing response force, is employed on the assumption of a lack of coupling between the bearing control axes. For one control axis:

$$
\mathrm{F}_{\mathrm{x} \operatorname{lin}}=\mathrm{K} x \mathrm{x} \mathrm{xac}+\mathrm{Cxx} \mathrm{vx}
$$

A difference between the non-linear magnetic bearing response force in a given axis $F_{x m a g}$ ac, known on the basis of model calculations and its linear form $F_{x}$ ac lin determined by the formula:

$$
\mathrm{F}_{\mathrm{x} \text { lin }}-\mathrm{F}_{\mathrm{xmag}} \mathrm{ac}=\Delta \mathrm{F}
$$

in such a way that $\Sigma \Delta F^{2}=\min$ is sought with the least squares method. Thus, the linearized coefficients of stiffness and damping $\mathrm{Kxx}_{\mathrm{x}}[\mathrm{N} / \mathrm{m}], \mathrm{Cxx}_{\mathrm{x}}[\mathrm{Ns} / \mathrm{m}]$ are obtained.

Changes in the non-linear magnetic response force $F_{m x}$ ac, provided by the digital controller for a given control axis, which result from the numerical calculations, are approximated with the linearized harmonic time history $F_{x}$ ac lin. Its values are determined by the dynamic stiffness $K x x$ and damping coefficients $C_{X x}$ for a given control axis. An analogous situation refers to the coefficients $K_{Y Y}, C_{Y Y}$ for the axis $Y$.

It is possible to use the proposed method for calculation of dynamic coefficients of the bearing when the developed simulation model of the bearing, whose operation is convergent with the operation of a real bearing system, is employed.

The calculations are conducted for stable bearing operation, where the journal position oscillates around the assumed point of equilibrium and the interactions between the control axes $X$ and $Y$ can be neglected for small displacements of the journal (Figure 14).
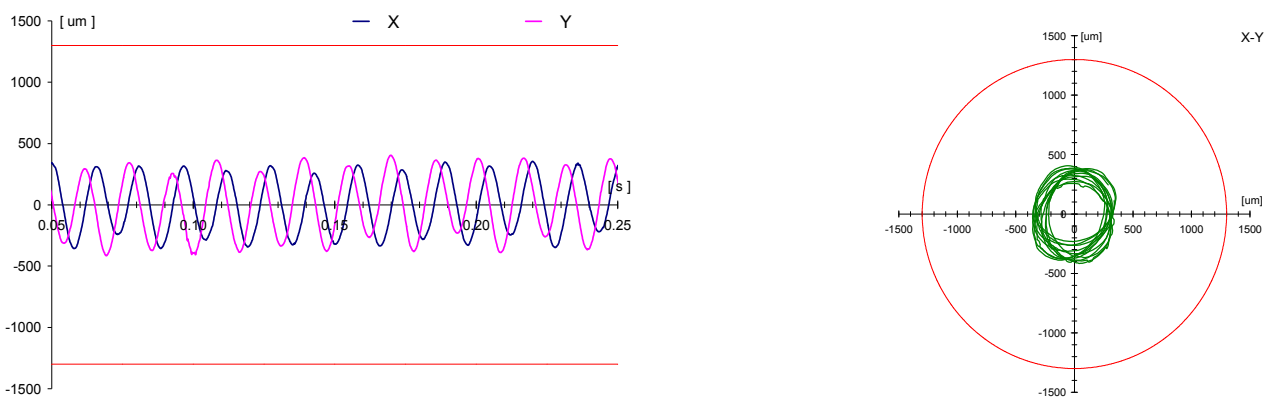

Figure 14. Displacements $X, Y$ versus time and the orbit

Figure 15 presents a comparison between the measured magnetic response force Fxmag and the theoretical function, which is a sum of the forces of stiffness and damping $F \times \operatorname{lin}=K \times x x+$ CXx Vx.

The curve $F x$ lin has been plotted on the basis of the measured journal displacement $x$ (Figure 14) and the journal velocity $V x$ obtained through digital differentiation of the displacement and a selection of suitable values of the dynamic stiffness coefficients $K x x$ and the damping 
coefficients $C_{x x}$ in such a way as to make the sum of squares of differences minimal for the selected part of the time history.

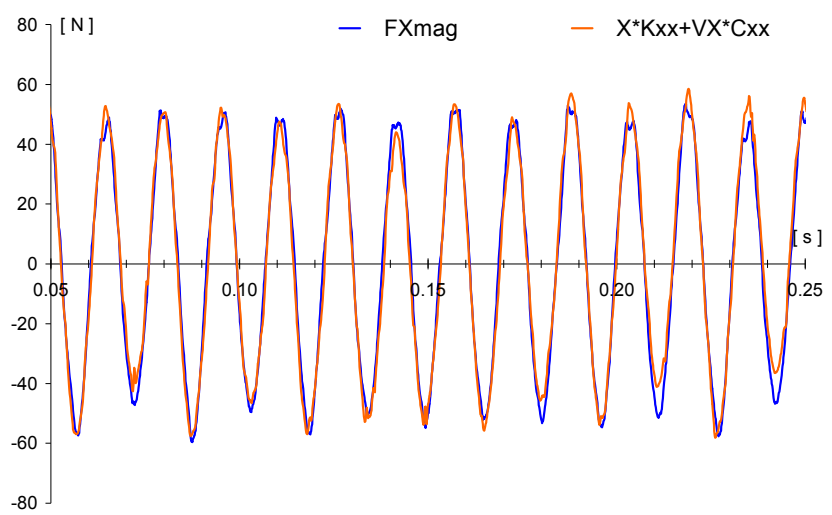

Figure 15. Measured magnetic response component along the control axis $X-F_{X m a g}$ and its modelled time history $F_{x}$ lin with the identified dynamic coefficients $K_{x x}, C_{x x}$

In the method of identification of bearing dynamic coefficients, it is required that the theoretically calculated magnetic response force is the closest approximation of its function obtained in the measurements and that the share of synchronous components in the curves of displacement, current and magnetic response force is dominant $[5,6,9]$.

\section{Results of the investigations}

The model test stand of the flexible power-transmission shaft is used to carry out the experiment whose aim is to verify the identification procedure of dynamic coefficients of the active magnetic bearing (Figure 16). The test rig consists of the horizontal shaft supported in two rolling bearings mounted at both ends. It is driven by an electric motor connected to the shaft through an elastic membrane coupling with smooth rotation control and fed by a frequency converter. An active magnetic bearing operates as an auxiliary bearing that modifies the dynamic properties of the shaft line. Between the magnetic bearing and the shaft right end, there is a rigid disk which allows one to mount balancing weights for the real structure. The mass of the rotating system is equal to $4.85 \mathrm{~kg}$, the shaft line length equals $1923 \mathrm{~mm}$. The test stand allows one to investigate the effects of the magnetic bearing on dynamic properties (vibration level, displacement and the coefficient of vibration amplification of subsequent critical frequencies) and to control vibrations of the long, flexible rotor. A kinematics exciter is fixed on the disk, on which masses of test unbalancing can be mounted. After introducing a selected program for magnetic bearing control, harmonic vibrations of the shaft of the frequency $(10,20,30, \ldots \div 80) \mathrm{Hz}$ and the assigned amplitude were excited. For each frequency under analysis, the time histories of displacements and currents in the magnetic bearing, which were subject to respective calculation procedures, were recorded, and then the bearing dynamic parameters were 
estimated. To conduct the measurement and calculation procedures, a measurement system with $D B K 15$ input systems made by IOtech, operating with a PC and employing the Daq/112B type PCMCIA measurement card of the resolution equal to 12 bites and the maximum sampling frequency of $100 \mathrm{kHz}$, was applied.

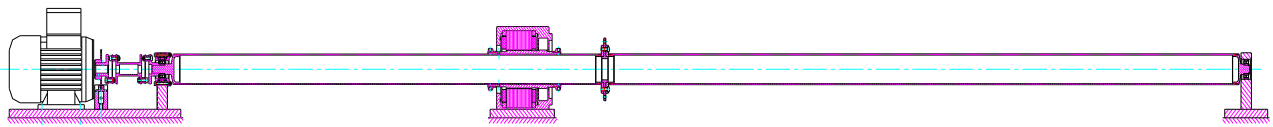

Figure 16. Configuration of the test rig

The voltage time histories corresponding to displacements (positions) of the journal along both the control axes $X, Y$ were recorded on-line on respective inputs of the measurementcontrol module. These were two voltage signals $0-24 \mathrm{~V}$ from Bently-Nevada type 3300 eddycurrent transducers of relative vibrations. The voltage time histories corresponding to currents flowing in electromagnets were measured and recorded. These were four voltage signals $0-5 \mathrm{~V}$ from current-voltage LEM type transducers.

The DaqView v.7.9.8 software was used for recording purposes. There were 4000 measurements made, at the sampling frequency of $8 \mathrm{kHz} /$ channel. The results were stored in binary files of the data acquisition system, and then converted into text files. The programs for analysis of dynamics and identification procedures of bearing dynamic parameters, according to the methodology proposed, were developed with the MS Excel spreadsheet.

Exemplary time histories of the quantities measured are shown in Figures 17 and 18 and of those calculated - in Figures 19 and 20 for the magnetic bearing of the selected configuration of the control program, at the kinematic excitation of the frequency $40 \mathrm{~Hz}$ and the assigned amplitude, whose value was such as to obtain the dominant share of synchronous components in the time histories under analysis and to obtain the linear range of magnetic response forces.
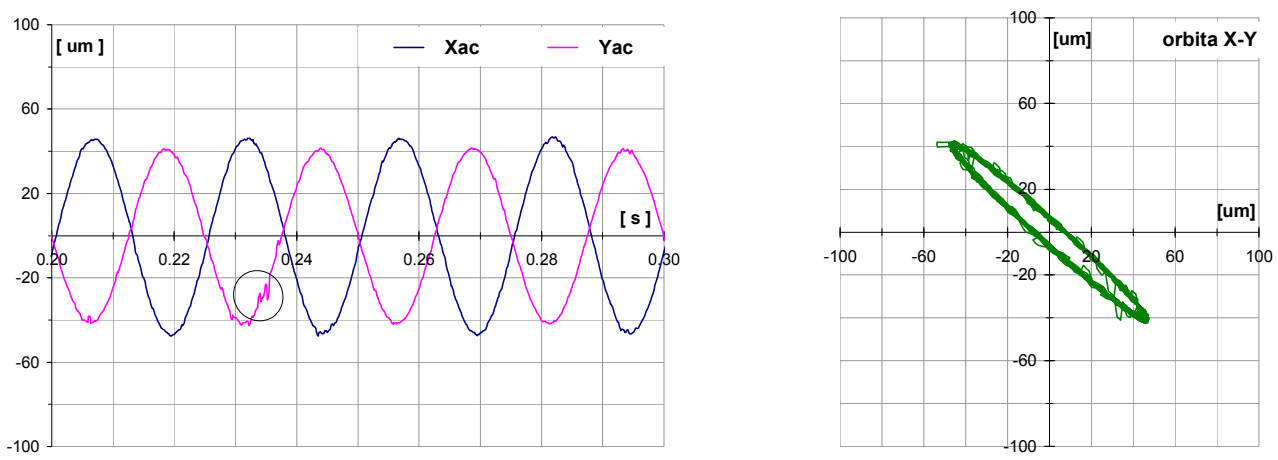

Figure 17. Displacements for both the control axes $X, Y$ and the shaft motion trajectory

The occasional disturbances which occur in the recorded time histories of displacements (Figure 17) are amplified by the digital differentiation and the effect of these disturbances is 
very distinct in the time history of the velocity component $V_{Y}$ (Figure 19a). This does not affect the calculation accuracy, where the characteristics are approximated with the harmonic time history.

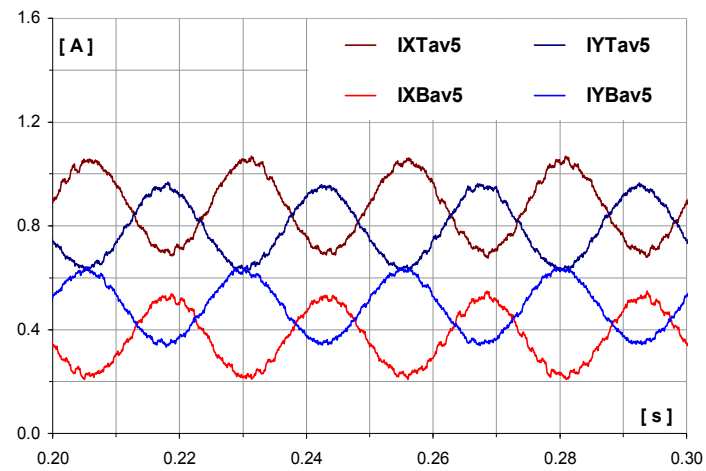

Figure 18. Time histories of the currents in electromagnet windings $І_{х т}, I_{х B}, I_{Y T}$, IҮв - averaged

a

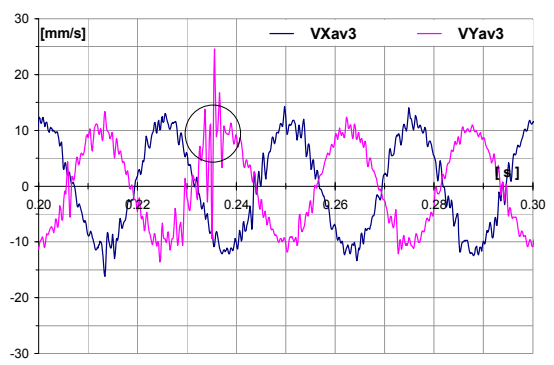

$b$

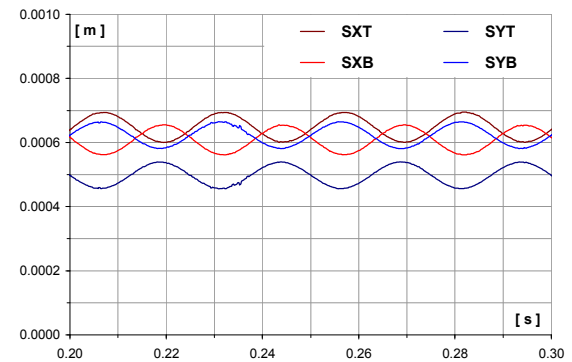

Figure 19. a. - Velocity components for both the control axes $V_{x}, V_{Y}$ - averaged b. - Variable gaps for individual electromagnets $X T, S_{X B}, S_{Y T}, S_{Y B}$

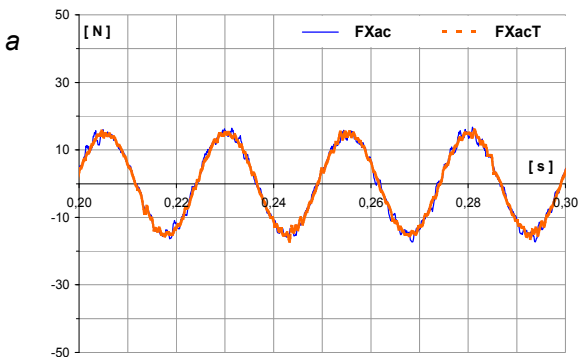

$b$

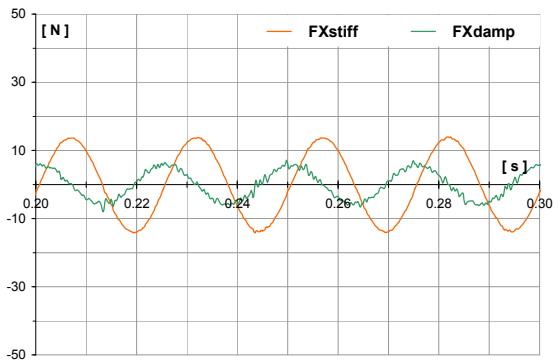

Figure 20. a. - Measured magnetic response component $F_{X a c}$ and the force $F_{X a c T}=F_{X}$ lin modelled with the identified dynamic coefficients $K x x, C x x$

b. - Components of the magnetic response related to the stiffness Fxstiff and to the damping Fxdamp

The measurement and calculation cycles were conducted for various excitation frequencies in the range $(10 \div 80) \mathrm{Hz}$, which allowed one to build functions representing the values of the 
bearing dynamic coefficients, namely: the stiffness coefficients $K_{x x}, K_{r y}$ and the damping coefficients $C_{X X}, C_{Y Y}$, as a function of frequency at the given excitation frequency and the given configuration of the control program (Figures 21).

a

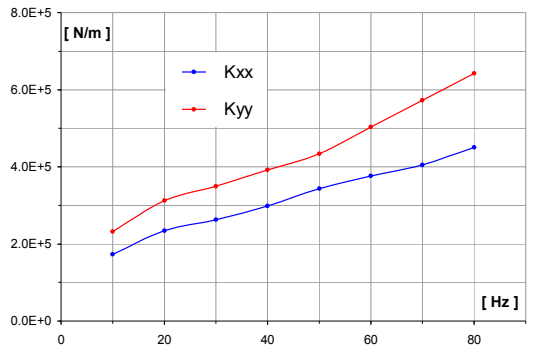

$b$

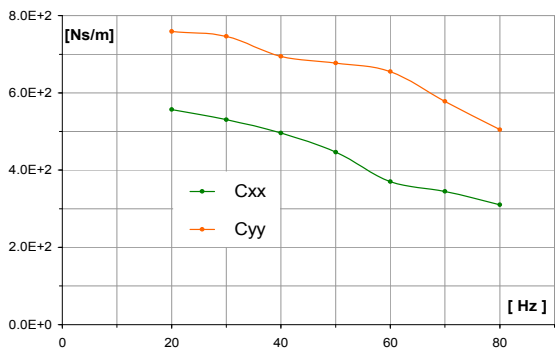

Figure 21. a. - Stiffness $K_{X X}, K_{Y Y}$ versus frequency b. - Damping $C_{X X}, C_{Y Y}$ versus frequency

It means that the start-up and shut-down characteristics of the model shaft line under such magnetic bearing operating conditions should be the overcritical characteristics. The experimentally determined dynamic coefficients of the bearing show some anisotropy of the properties for individual axes $K_{X x} \cong K_{Y Y}, C_{X X} \cong C_{Y Y}$. The analysis of this state showed that for isotropic properties of the energy transmission systems (symmetrical saturation-control current characteristics), this scattering resulted from the scattering of constant values of electromagnets for the given axis. This conclusion was confirmed by the calculations conducted with the simulation model of the magnetic bearing.

The experiment was conducted for various configurations of the program controlling the auxiliary magnetic support used in the model shaft line system. The determined dynamic coefficients were employed in subsequent stages of the investigations in modelling and numerical calculations of start-up and shut-down characteristics of the flexible powertransmission shaft. The generated numerical characteristics were verified experimentally through a comparison to the start-up and shut-down curves recorded for the real model shaft line with supports of identified dynamic properties.

The proposed methodology of measurement of response and dynamic coefficients of the magnetic bearing is a very important tool in designing dynamics and vibration control of machine rotors in which active magnetic bearings are applied. It allows one to find analogies to classical bearing systems and to employ professional calculation codes for evaluation of the effects of modification in the dynamic properties of shaft lines introduced through changes in the configuration of the program controlling its active magnetic supports [4-9].

\section{Modification of the dynamics of the rotating system}

The last step of this work is to present the experimental results of maintaining a low level of vibrations in the whole operating range of the flexible rotor by the application of an 
auxiliary active magnetic bearing where the biggest anticipated dynamic deflection of the rotor occurs.

The application of an auxiliary magnetic bearing to modify the dynamics of the rotating system demands the comparison of the classical (without a magnetic bearing) and modified design. In the proposed construction of the test stand, a flexible rotor is supported in ball bearings. An electric engine integrated with the shaft is used as a drive (Figure 22).

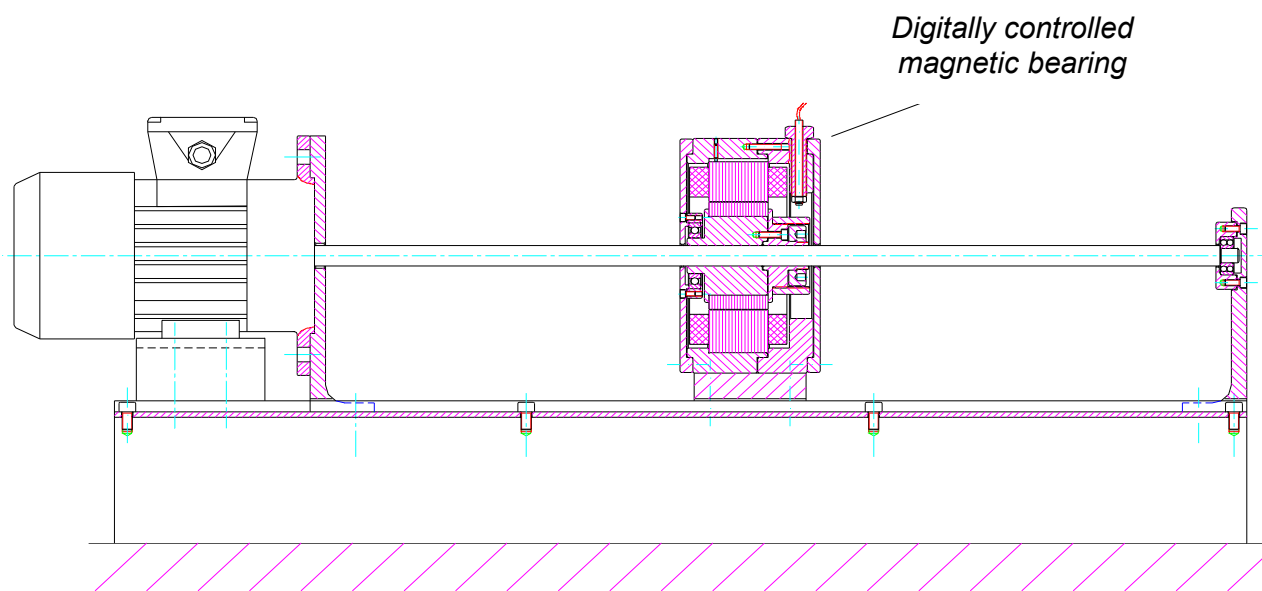

Figure 22. Scheme of the rotating system with an auxiliary active magnetic bearing

The length and the diameter of the shaft is $700 \mathrm{~mm}$ and $17 \mathrm{~mm}$, respectively. Each bearing support is connected to the foundation by rigid elements. The mechanical parameters of the designed magnetic bearings are as follows:

- $\quad$ diameter of the journal $89.5 \mathrm{~mm}$

- $\quad$ radial clearance $0.5 \mathrm{~mm}$

- number of electromagnet coil pairs4

- length $45.9 \mathrm{~mm}$

- $\quad$ static load $300 \mathrm{~N}$

- two axes of control $x, y$ with eddy-current transducers.

In this design, the feasible assembly and disassembly of the auxiliary magnetic bearing is assumed. It is the position of the auxiliary magnetic bearing, mounted on the shaft of the test stand, which allows one to model the mode of lateral vibrations. A numerical experiment shows the usefulness of this concept in the case of the test stand rotor. However, the practical application of the presented idea demands serious experimental verification. The aim of this work is to present the results of the experimental investigations of the dynamic response of the system to the synchronous excitation corresponding to the unbalance occurring in the centre of the rotor. 
In order to modify the dynamic properties of this structure, an additional journal magnetic bearing should be introduced. The following two cases are considered:

- flexible rotor supported in classical (ball) bearings - the magnetic bearing turned off,

- flexible rotor supported in classical (ball) bearings and an additional magnetic bearing the magnetic bearing turned on.

When a machine is supported in the whole system of magnetic bearings, the bearings carry the main load that comes from the rotor (lateral, thrust). The role of the magnetic bearing in the system under consideration is to introduce an additional instantaneous point of support for the rotor and to carry a part of the dynamical (lateral) load. Thrust load is always carried by classical (ball) bearings. The proposed additional magnetic bearing does not introduce any additional thrust load. That is why in the proposed analysis, the effect of the active magnetic bearing on thrust force acting on the rotor system isn't under consideration.

The operation of an auxiliary magnetic bearing in the rotating system just before a high level of the amplitude is achieved, causes a qualitative change in dynamic properties of the system. It corresponds to the critical frequency of lateral vibrations and allows for reaching the nominal revolutions of the machine without dangerous effects connected with exceeding the critical frequencies of the flexible rotor.

\section{Numerical calculations and verifications}

The test stand as presented in Figure 22 was modelled numerically. A professional program $D Y R O B E S$ that allows for modelling the dynamics of the shaft line of rotary machines was employed in the numerical calculations. Numerical calculation methods allow one to carry out a complete analysis of machine rotor vibrations. The geometry of the rotating system was modelled with discrete elements. The magnetic bearing journal is situated in the centre of the shaft line (Figure 23).

In Figures 23a and b a shaft line model and calculated modes of critical frequencies of the rotating system for two configurations, i.e. when the magnetic bearing is turned off and when it provides an additional support of the stand, are depicted.

In the configuration under consideration, the dynamic properties of the magnetic bearing are a vital element that decides about a value of the critical frequency of the analysed shaft line. These properties are connected with the assumed parameters of the control system and were identified through the analysis of the bearing simulation model.

The analysis of the rotating system dynamics was performed and the first lateral critical frequency and its respective deflection line of the rotor supported in ball bearings were determined $(38.2 \mathrm{~Hz})$. The required value of the dynamic stiffness of the magnetic bearing that allows for avoiding the necessity of exceeding the critical value at the start-up of the model shaft line is equal to $K_{M}>3 E+5 \mathrm{~N} / \mathrm{m}$.

The second stage of calculations consisted in a determination of the theoretical start-up curve of the modelled shaft line of the test stand. After introducing the linearized 
coefficients of stiffness and damping of the auxiliary active magnetic bearing, determined on the basis of the model, a response to the rotating synchronous excitation was determined.

$\boldsymbol{a}$

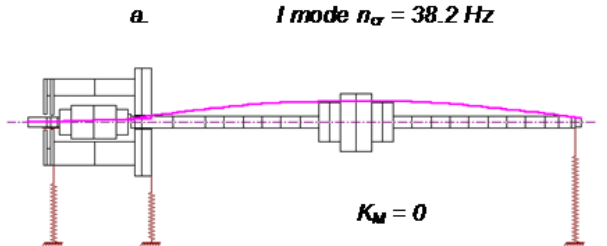

b.

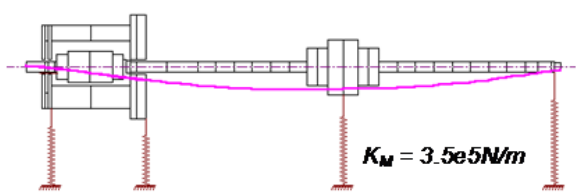

Figure 23. Shaft line model of the test stand and the first critical mode of vibrations a) magnetic bearing turned off, b.) magnetic bearing turned on

The synchronous excitation with a force coming from the residual unbalance was introduced. The values of the vibration amplitude as a function of frequency are the system response. For the first critical frequency, the vibration amplitude reaches the value of $260 \mu \mathrm{m} p-p$ for the rotating system supported only in ball bearings (Figure 26b). After introducing the auxiliary magnetic bearing in the form of dynamic coefficients of stiffness and damping into the model calculations, the theoretical Bode plot shown in Figure 26 was obtained.

The experimental investigations, like the theoretical ones, were carried out in two stages:

- $\quad$ with a fixed shaft of the test stand,

- during the start-up and shut-down of the model rotating system.

In the first stage, the dynamics of the rotating system was analysed by means of the modal testing method.

For a fixed shaft of the test stand, the tests were carried out for two variants of operation: with the magnetic bearing turned on and turned off.

The system was forced to vibrate by means of the excitation pulse force, whose direction was convergent with the direction of the response measurement (selected control axis). The spectral concentration of this excitation in the frequency range investigated $0 \div 200 \mathrm{~Hz}$ was practically constant.

The analysis of the time histories (Figure 24a) points out to the fact that in the system with the magnetic bearing turned off, weakly damped oscillations occur as a result of the excitation and they disappear after approximately $t=3 \mathrm{~s}$.

In the system in which the bearing is activated, these vibrations are strongly damped. In the whole time span observed, slight oscillations of the rotor occur. They result from the principle of bearing operation, which consists in the continuous control of the assumed position of the rotor balance. The time histories were recorded in the time interval $t=4 \mathrm{~s}$ in order to provide evidence of stable operation of the system after the pulse excitation force had been applied. 
A

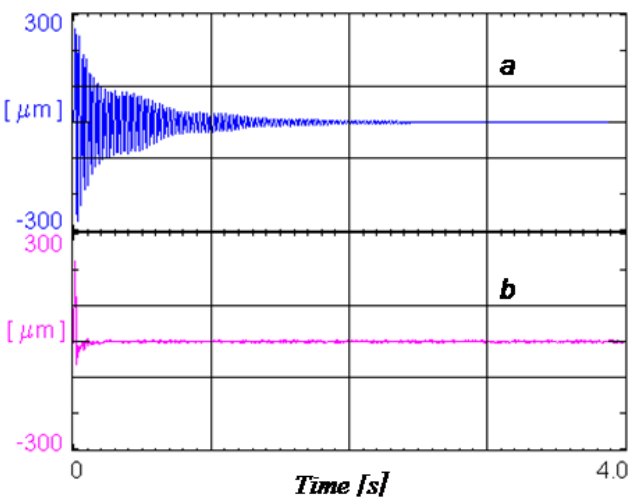

B

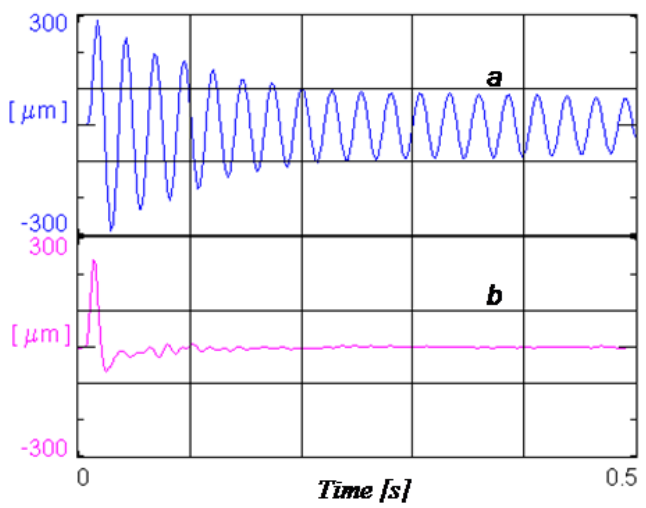

Figure 24. Time history of the response (displacement) recorded along the direction $x$ for the fixed rotor $A$ - recording time $t=0 \div 4 \mathrm{~s}, B$ - recording time $t=0 \div 0.5 \mathrm{~s}$ a.) auxiliary magnetic bearing turned off, $\mathrm{b}$.) auxiliary magnetic bearing turned on

To show the dynamics of the response of the system with the activated magnetic bearing in Figure $24 \mathrm{~b}$, the same time histories of the response are presented for the shorter recording time.

During this time, an effect of the system modification that consists in an activation of the additional magnetic bearing with the programmable assigned dynamic properties is clearly visible. Then, the shaft vibrations disappear after approximately $t=0.05 \mathrm{~s}$.

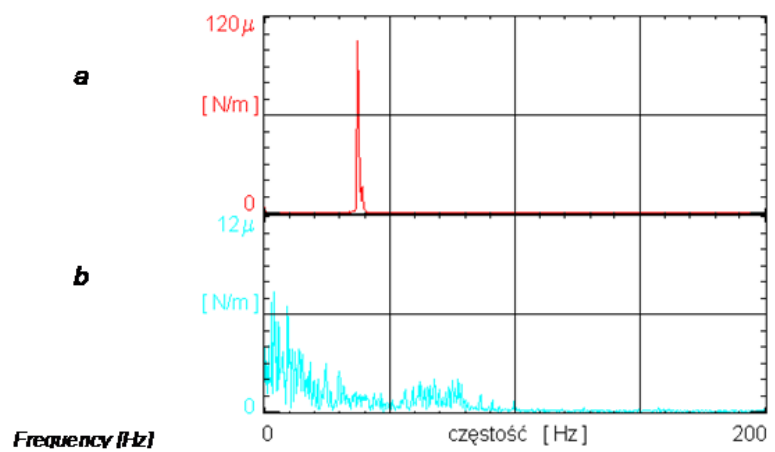

Figure 25. Frequency response for the axis $x$ in the frequency range $n=0 \div 200 \mathrm{~Hz}$ for the fixed rotor a) auxiliary magnetic bearing turned off, b) auxiliary magnetic bearing turned on

In Figure 25 the transmittances of the rotating system measured on the magnetic bearing journal have been compared.

The analysis of these functions shows that in the system with the bearing turned off, weakly damped vibrations with the frequency of approximately $n=39 \mathrm{~Hz}$ (the first vibration frequency of the rotor in this configuration - Figure 26a) dominate. 
The transmittance of the system with the bearing turned on exhibits strong vibration damping. The applied pulse excitation is not able to generate self-vibrations of the rotor. The recorded frequency response (Figure $25 b$ ) is located on the level of noises and equals $\approx 6 \mu m$. The first frequency of self-vibrations of the rotor in this configuration (estimated in the calculations to be equal to approx. $n=60 \mathrm{~Hz}$ - cf. Figure $26 \mathrm{~b}$ ) does not occur in practice in the results of the experimental investigations. The investigations carried out when the rotor did not move have proven a significant influence of the auxiliary magnetic bearing on rotor dynamics.

They have shown possibilities of obtaining an abrupt change in the dynamic properties of the rotating system, which complies with the idea of the safe exceeding of the critical frequency of the machine.

In the second stage of the experimental investigations, the dynamics of the model rotating system with a synchronous excitation with unbalance forces was analysed. The investigations were carried during the start-up and shut-down of the system rotor. Numerical simulations of the test stand rotor response to unbalance were performed. An example of the calculation results shown in Figure 26 indicates that for the nominal rotational frequency $50 \mathrm{~Hz}$, the test stand rotor can be an overcritical or undercritical system, depending on the fact whether the auxiliary magnetic bearing characterised by specified dynamic properties is activated or disactivated.
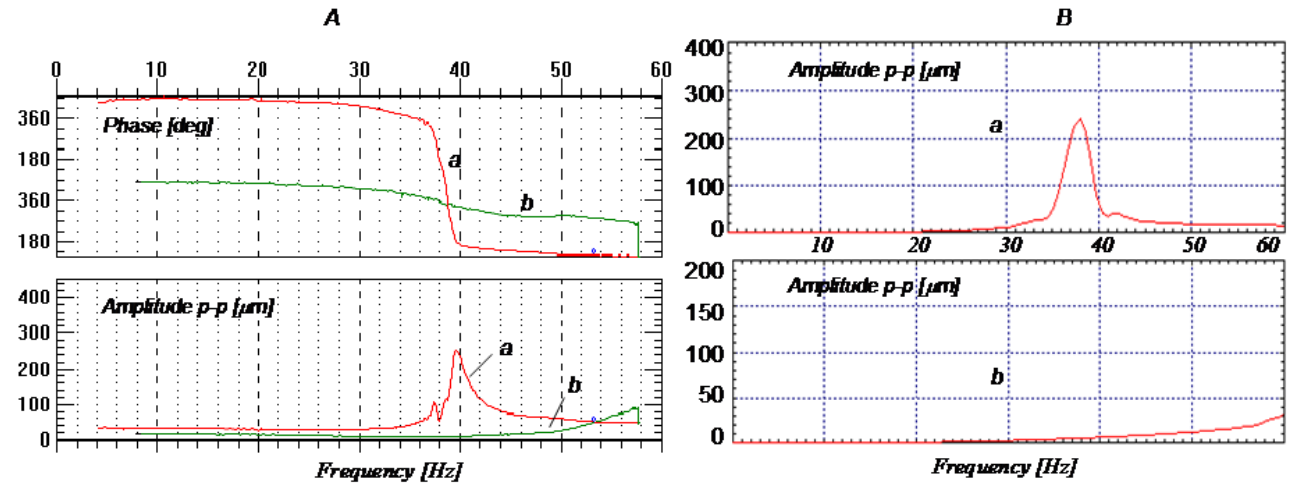

Figure 26. $A$ - Experimental Bode plot $B$ - Theoretical Bode plot $a$-start-up, the rotating system supported in ball bearings, $b$ - shut-down, with the auxiliary magnetic bearing activated

An application of the auxiliary magnetic bearing allowed for a complete elimination of a dangerous increase (for the whole local system operation) in the vibration amplitude when the critical frequency had been exceeded. The optimal moment for activation and disactivation of the additional bearing system has been indicated by a solid line. At this moment, the vibration amplitudes of both the qualitatively different dynamic systems reach similar values. In the presented example, the switching took place above the optimal values of revolutions, which was connected with an abrupt change in the vibration amplitude and phase. 
In Figure 27 cascade plots of the journal vibrations in the magnetic bearing during the startup of the test stand in the so-called "full spectrum" domain are presented. A comparison of the diagrams illustrates the role played by a presence of the auxiliary magnetic bearing from the point of view of shaft vibrations during the start-up (or shut-down) of the model machine.
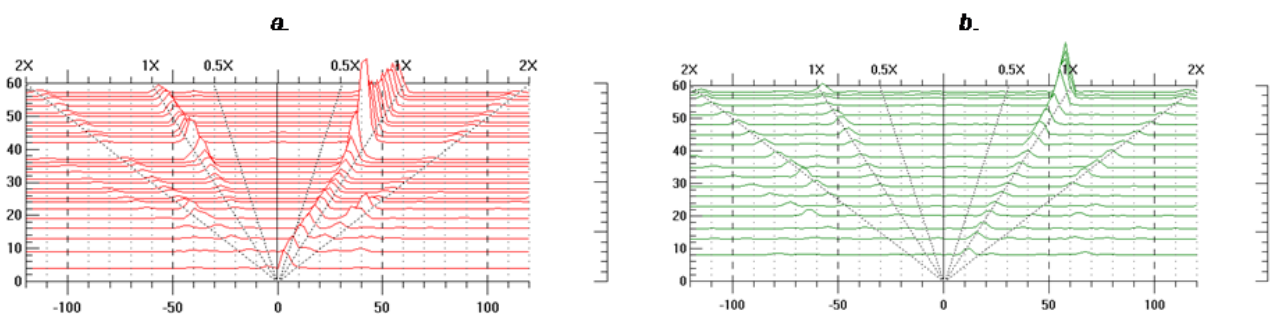

Figure 27. Cascade plots of the journal vibrations in the magnetic bearing during the start-up of the model shaft line a) auxiliary magnetic bearing disactivated, b) auxiliary magnetic bearing activated

The presented idea of an application of the active magnetic bearing as a system that modifies the dynamic properties of the rotating system can turn out to be an interesting alternative to modernisation of a real rotary machine. The majority of overcritical failures of rotors is caused by a local increase in the synchronous or asynchronous vibration amplitude and by exceeding the clearances when the critical frequency or the stability threshold of the rotating system is exceeded. The special character of the active magnetic bearing operation makes it possible to activate it when the shaft rotates, whereas relatively high values of the clearances allow for locating it practically in any place of the machine shaft line. The built test stand enabled practical realisation of the idea of "omitting" the region in which rotor vibrations, dangerous for the machine operation, appear.

\section{Conclusions}

The progress in the field of active magnetic bearings is based on new operating and diagnostic capabilities of these digitally controlled bearings in comparison to classical solutions. The overall goal of an $A M B$ controller is to stabilise the plant and to reach the optimal technical performance. To achieve these goals, $A M B$ systems have to be optimised in an overall mechatronics design approach. This leads to a new concept for control systems and actuators [11-14].

In this work, an idea of the simulation model of magnetic levitation systems and its diagnostic capabilities is presented. Some results of the numerical simulations and experimental investigations are discussed. 
The conducted investigations allowed one to verify experimentally stiffness and damping of the real rotor-magnetic bearing system by means of a numerically calculated model of the rotor.

The proposed methodology of measurement of response and dynamic coefficients of the magnetic bearing is a very important tool in designing dynamics and vibration control of machine rotors in which active magnetic bearings are applied. It allows one to find analogies to classical bearing systems and to employ professional calculation codes for evaluation of the effects of modification in the dynamic properties of shaft lines introduced through changes in the configuration of the program controlling its active magnetic supports.

A comparison of the theoretical time histories with those obtained experimentally confirms the correctness of the proposed method for the determination of dynamic coefficients of the magnetic bearing. Achieving the nominal speed of the flexible rotor and maintaining a low level of vibrations in the whole operating range of the rotating system (including critical speeds) by using an auxiliary active magnetic bearing is a very interesting idea for the rotating machinery.

The operation lets "omit" the zone of a dangerous increase in the amplitude of rotor vibrations, which is connected with the critical speed of lateral vibrations. The experiment shows the usefulness of this concept in the case of the real rotating system.

The symmetry of characteristics of individual actuator paths of bearings, which has been programmable corrected and experimentally verified, has made it possible to implement an idea of the application of a single controller to control the real journal bearing operation along both axes. A selection of values of controller parameters is based on the investigation of the simulation model of an active magnetic bearing system.

The procedures of numerical representations of the actuator characteristics have allowed for a development of the model whose operation is convergent with the real bearing system. It enables simulation investigations of the dynamics of the mass suspended in the bearing bush under widely variable values of controller parameters and under various disturbances and forces. A reliable theoretical model that allows for analysis of the bearing dynamics under hypothetical, extreme loads reduces the designing time and enables one to minimize errors that can occur at the system prototype start-up.

The experimental characteristic curves of the start-up and shut-down have confirmed possibilities of the programmable modelling of dynamics of the shaft line, and - in prospect of the designed machine that includes an additional active magnetic bearing.

\section{Author details}

Dorota Kozanecka

Institute of Turbomachinery, Lodz University of Technology, Łódź, Poland 


\section{References}

[1] Schweitzer G., Traxler A., Bleuler H., (1993), Magnetlager, Springer-Verlag, Berlin (in German).

[2] Kozanecka, D., et al., (1997), Application of an Auxiliary Active Magnetic Bearing for Modification of Dynamic Properties of Rotors, Proc. of the XIV World Congress IMEKO, June, Finland, Vol. IX, pp. 93-98.

[3] Delprete, C., Genta, G., Repaci, A., (1998), Numerical Simulations of the Dynamic Behaviour of Rotors on Active Magnetic Bearing, Proceedings of the 7 Int. Symp. on Transport Phenomena and Dynamics of Rotating Machinery, Honolulu, Hawaii, February, Vol. A, pp. 48-57.

[4] Kozanecka D., (1999), Dynamic Properties of the Rotor Magnetic Suspension System, Symkom'99 Łodz, Technical University of Łodz, Journal CMP - Turbomachinery, No 115 , pp. 217-224.

[5] Kozanecka D., 2001, Dynamic of the Flexible Rotor with an Additional Active Magnetic Bearing, Machine Dynamics Problems, 2001, Warsaw, Vol. 25, No. 2, pp. 21-38.

[6] Kozanecka D., Kozanecki Z., 2001, Modelling the Dynamics of Active Magnetic Bearing Actuators, Proceedings of the World Multiconference on Systemics, Cybernetics and Informatics, SCI 2001, July 22-25, USA, Vol. IX, Industrial Parts I, pp. 232-235.

[7] Kozanecka D., et al., 2003, New Concept of the Spin Test System with Active Magnetic Bearing, Procds. $2^{\text {nd }}$ International Symposium on Stability Control of Rotating Machinery, ISCORMA, Gdansk 4-8 August 2003, Poland, pp. 199-219.

[8] Kozanecka D., et al., (2007), Application of Active Magnetic Bearings for Identification of the Force Generated in the Labyrinth Seal, Journal of Theoretical and Applied Mechanics, Polish Society of Theoretical and Applied Mechanics, No.1, Vol. 45, pp. 53-60.

[9] Kozanecka D., et al., (2008), Experimental Identification of Dynamic Parameters for Active Magnetic Bearings, Journal of Theoretical and Applied Mechanics, Polish Society of Theoretical and Applied Mechanics, No.124, Vol. 46, pp. 41-50.

[10] Schweitzer G., Maslen E.H., (2009), Magnetic Bearings. Theory, Design and Application to Rotating Machinery, Springer-Verlag, Berlin, Heidelberg.

[11] Gizelska M., Kozanecka D., Kozanecki Z., (2009), Integrated Diagnostics of the Rotating System with an Active Magnetic Bearing, Solid State Phenomena, Trans Tech Publications Ltd, Switzerland, ISSN 1012-0394, Vol. 147-149 pp 137-142. (Online available since 2009/Jan/06, at http://www.scientific.net)

[12] Łagodziński J., (2009), Modeling of Magnetic Fields with the Finite Element Method in Machine Diagnostic Systems, Solid State Phenomena, Trans Tech Publications Ltd, Switzerland, ISSN 1012-0394, Vol. 147-149, pp. 155-160. (Online available since 2009/Jan/06, at http://www.scientific.net)

[13] Kozanecka D., Kozanecki Z., Łagodziński J., (2011), Active Magnetic Damper in a Power Transmission System, Communications in Nonlinear Science and Numerical Simulation, Journal Elsevier, No 16, pp. 2273-2278 www.elsevier.com/locate/cnsns_1567. 
[14] Kozanecka D., (2010), Diagnostics of Rotating Machinery Mechatronic System, Monographic Series of Publications: Maintenance Problems Library, Scientific Publishing House of Institute for Sustainable Technologies in Radom, ISBN 978-83-7204 966-7, (in Polish). 